gerer Entwicklungen aufdrängen. So wäre es wünschenswert gewesen, die Bedeutung informeller Verfahren für die Gesetzgebung - gerade vor dem Hintergrund einer wachsenden Tendenz zu frühzeitigen Einigungen mit dem Rat - sowie die Konsequenzen für den Parteienwettbewerb näher zu beleuchten. Dies gilt umso mehr, als Ringe in seinem Literaturüberblick eine Reihe extrem interessanter Arbeiten ${ }^{1}$ zu diesem Thema aufführt und diesen Aspekt auch in einer der Fallstudien anreißt. Unberücksichtigt bleibt auch die zunehmende Praxis arbeitsteiliger Verfahren zwischen den Ausschüssen. Hierbei haben Berichterstatter des federführenden Ausschusses vor allem in der Frühphase des Gesetzgebungsprozesses nicht mehr so freie Hand in der Steuerung der parlamentarischen Beratung. Noch relevanter ist die Tatsache, dass Ausschüsse teilweise umgangen werden zugunsten informeller Foren, in denen vor allem Experten der beiden großen Fraktionen - Sozialdemokraten und Christdemokraten - eine maßgeblich Rolle für die Konsensbildung und Entscheidungsfindung spielen. ${ }^{2}$ Diese auch demokratietheoretisch wesentlichen Aspekte der Verfahrenssteuerung näher zu untersuchen muss folglich zukünftigen Arbeiten vorbehalten bleiben.

Nichtsdestoweniger ist Nils Ringe mit „Who Decides, and How?" eine theoretisch wie methodisch überzeugende und anspruchsvolle, zugleich anschauliche und gut lesbare Monographie gelungen, die dem Forschungsstand zum EP im Speziellen und zur Parlamentsforschung im Allgemeinen Substanzielles hinzuzufügen vermag, weil sie ihr zentrales Anliegen einlöst: eine Erklärung für die Ursachen hoher fraktioneller Geschlossenheit zu liefern, die am individuellen Abgeordneten und seiner Entscheidungsfähigkeit ansetzt.

Stefan Thierse

1 Vgl. Henry Farrell / Adrienne Héritier, Interorganizational Negotiation and Intraorganizational Power in Shared Decision Making: Early Agreements Under Codecision and Their Impact on the European Parliament and Council, in: Comparative Political Studies, 37. Jg. (2004), H. 10, S. $1184-1212$.

2 Vgl. Pierpaolo Settembri / Christine Neuhold, Achieving Consensus Through Committees: Does the European Parliament Manage?, in: Journal of Common Market Studies, 47. Jg. (2009), H. 1, S. $127-151$.

\title{
Politikfelder der Europäischen Union: ideale Lektüre für Einsteiger
}

Pollack, Johannes, Samuel Schubert und Peter Slominski: Die Energiepolitik der EU (Reihe Europa Kompakt, Band 5), Facultas, Wien 2010, 232 Seiten, € 19,50.

Puetter, Uwe: Die Wirtschafts- und Sozialpolitik der EU (Reihe Europa Kompakt, Band 3), Facultas, Wien 2010, 272 Seiten, € 19,50.

In den letzten Jahren wurden zahlreiche Bücher veröffentlicht, die sich mit der Europäischen Union (EU) befassen. Die meisten dieser Untersuchungen widmen sich der Frage, wie sich die institutionellen Strukturen auf Entscheidungssituationen auswirken beziehungsweise wie sich das europäische Mehrebenensystem auf die Politikgestaltung auswirkt. Vor diesem Hintergrund stellen die hier zu besprechenden Bücher aus der Reihe „Europa 
Kompakt" eine durchaus willkommene Abwechslung dar, da sie die verschiedenartigen Politikfelder der EU auf eine umfassende Art und Weise einer breiten Leserschaft näher bringen wollen. Dabei fällt auf, dass sich die Autoren Politikfeldern widmen, die sich recht unterschiedlich entwickelt haben. Pollack u.a. arbeiten die zentralen Merkmale der gemeinschaftlichen Energiepolitik heraus, die erst in jüngerer Vergangenheit mit dem Grünbuch der Kommission „Eine europäische Strategie für nachhaltige, wettbewerbsfähige und sichere Energie" ein gewisses Maß an Kohärenz gewonnen hat und noch weiterentwickelt werden muss, um den vielfältigen energiepolitischen Herausforderungen zu begegnen. Puetter bietet eine Einführung in die europäische Wirtschafts- und Sozialpolitik, zwei Politikfelder, die zwar eng miteinander verschränkt, aber hinsichtlich des Vergemeinschaftungsgrades recht unterschiedlich sind.

Pollack u.a. beginnen ihre Ausführungen mit einer umfassenden Einführung in die technischen und ökonomischen Grundlagen der europäischen Energiepolitik. Das Kapitel vermittelt einen kompakten und informativen Eindruck hinsichtlich der Energieerzeugung und des Energieverbrauchs in der EU. Zahlreiche Tabellen und Graphiken erlauben dem Leser einen unmittelbaren Einstieg in die Thematik und regen dazu an, die Varianz zwischen den Mitgliedstaaten näher zu untersuchen. Anschließend wird die Entwicklung des Politikfeldes im Zeitverlauf näher beschrieben. Die Autoren erläutern, wie wichtig Energiefragen zu Beginn des Integrationsprozesses waren, da dieser schließlich mit der Gründung der Europäischen Gemeinschaft für Kohle und Stahl sowie der Schaffung von Euratom begonnen hat. Ab den 1970er Jahren wurde der Gegenstandsbereich von Energiepolitik insofern breiter, als Fragen nach der Versorgungssicherheit und ab den 1980er Jahren Überlegungen zum Umweltschutz hinzu kamen. Im nächsten Schritt stellen die Autoren die wichtigsten energiepolitischen Akteure vor und geben einen Überblick über das primäre und sekundäre Energierecht. In diesem Zusammenhang legen sie den Schwerpunkt auf die Energieträger Elektrizität und Erdgas, da diese stärker von EU-Regelungen betroffen sind als Erdöl oder gar Atomenergie. Allerdings versäumen sie es auch nicht, die energiepolitischen Regelungen in einen Kontext mit der europäischen Umwelt- und Klimapolitik zu rücken. Das letzte inhaltliche Kapitel beschäftigt sich ausführlich mit dem außenpolitischen Handeln der EU im Energiebereich. In diesem Zusammenhang diskutieren Pollack u.a. Fragen der Effektivität des europäischen Regelexports (in der Forschungsliteratur auch unter dem Begriff „externe Governance“ bekannt) und der Nachbarschaftspolitik. Der Umfang dieses Kapitels legt dem Leser nahe, dass die Autoren vor allem in der außenpolitischen Dimension der europäischen Energiepolitik eine lohnenswerte analytische Perspektive sehen. Tatsächlich scheint diese implizite Einschätzung angesichts der Erdölabhängigkeit der Mitgliedstaaten gerechtfertigt. Allerdings stellt sich in diesem Zusammenhang die Frage, ob ein rein policy-analytischer Untersuchungsrahmen ausreichend ist oder ob nicht Theorien der Internationalen Beziehungen hierfür besser geeignet wären.

Insgesamt ermöglicht das Buch einen einfachen Einstieg in die komplexe Thematik der Energiepolitik. Die sprachliche Gestaltung und die Gliederung können als sehr gelungen bezeichnet werden. Ansprechend sind auch die zahlreichen Abbildungen sowie der Verweis auf einschlägige Ressourcen im Internet. Der Charakter des Buches als ein Einführungswerk ist ohne weiteres erkennbar: So ist es weitgehend beschreibend und nur selten analytisch. Auch wenn dies im Sinne der Reihe ist und explizit Hintergrund- und Kompaktwissen zum politikfeldspezifischen Integrationsprozess geboten werden sollen, wäre es vor allem bei der Darstellung der Akteure durchaus wünschenswert gewesen, theoretische Aus- 
sagen zu treffen. Allerdings muss den Autoren zugute gehalten werden, dass sie auf weiterführende Literatur verweisen. Angesichts des stark deskriptiven Charakters dürfte das Buch insbesondere für Studierende der Politikwissenschaft in einem früheren Studienabschnitt sowie Praktiker von Interesse sein.

Puetter beginnt seine Ausführungen mit einem Verweis auf die Relevanz von wirtschaftsund sozialpolitischen Aspekten für die Entwicklung eines gemeinsamen Marktes. In diesem Zusammenhang verdeutlicht der Autor, wie präsent das - jedoch nicht näher definierte europäische Sozialmodell in der politischen Rhetorik diverser europäischer Akteure ist. Puetter versucht diesem Prinzip analytische Schärfe zu verleihen, indem er unterschiedliche in der Forschungsliteratur verwendete Definitionen zusammenträgt und voneinander abgrenzt. Im Anschluss wird die Entwicklung beider Politikfelder illustriert, bevor der Autor die inhaltlichen Grundzüge sowie das umfangreiche Instrumentarium der europäischen Wirtschafts- und Währungspolitik vorstellt. Einleitend verweist er auf den Umstand, dass die Mitgliedstaaten zwar eine einheitliche Währungspolitik betreiben, die wirtschaftspolitischen Kompetenzen jedoch dezentral verortet sind. Hierbei verfolgt die Währungspolitik strikt das Ziel der Preisstabilität, während die Koordinierung der Wirtschaftspolitik in erster Linie auf die Haushaltsdisziplin der Mitgliedstaaten abzielt. Die zentralen Merkmale des wirtschaftspolitischen Koordinierungsverfahrens werden detailliert dargestellt. Des Weiteren diskutiert Puetter die wirtschafts- und währungspolitischen Konsequenzen aus der Erweiterung der Eurozone und dem Vertrag von Lissabon.

Die weiteren Ausführungen beschäftigen sich mit der Sozialpolitik, die im Vergleich zu anderen Politikfeldern zu einem eher geringen Grad vergemeinschaftet ist. So stehen in ihrem Mittelpunkt vor allem Regelungen zu Arbeitsmobilität, Arbeitszeit und -umgebung sowie die Gleichstellung von Männern und Frauen. Keine Kompetenzen besitzt die Gemeinschaft bei Fragen bezüglich sozialer Sicherungssysteme, Mindestsicherung, Armutsbekämpfung und sozialer Eingliederung, also den Kernbereichen der nationalen Sozialpolitiken. In Ergänzung zu seinen Ausführungen über Politikinhalte stellt Puetter die zentralen europäischen Entscheidungsorgane vor. Des Weiteren erläutert er die Organisation der europäischen Sozialpartner und erklärt, in welchem Umfang die Zivilgesellschaft in den Politikgestaltungsprozess eingebunden ist. Hierbei wird klar, dass die institutionalisierte Beteiligung von Arbeitgeberverbänden und Gewerkschaften die Partizipationsmöglichkeiten für Nichtregierungsorganisationen bisher spürbar eingeschränkt hat. Ob sich dies mit den erweiterten Beteiligungsmöglichkeiten auf der Grundlage des Vertrages von Lissabon ändern wird, lässt der Autor offen. Abschließend werden verschiedene Politikinstrumente vorgestellt, von denen die Gemeinschaft in diesen beiden Bereichen vornehmlich Gebrauch macht. Im Zentrum dieser Ausführungen stehen Regulierungen, wobei Puetter auch auf verschiedene Koordinierungsverfahren und Formen flexibler Integration zu sprechen kommt. Vor allem letzterer Aspekt ist von großer analytischer und praktischer Bedeutung, da es im Kern auf Strategien differenzierter Integration hinausläuft, die konkret auf eine uneinheitliche Anwendung gemeinschaftlicher Bestimmungen abzielen und somit das Potential für Desintegration bergen.

Puetter versteht es, auf eine relativ kompakte Weise die grundlegenden Charakteristika zweier komplizierter Politikfelder darzustellen. Die sprachliche Gestaltung sowie der Aufbau des Buches sind klar und machen es dem Leser leicht, in die Thematik zu finden. Der Band stellt eine ideale Lektüre für Studienanfänger und Praktiker dar, da er eine eindrückliche Beschreibung der inhaltlichen Ausgestaltung von europäischer Wirtschafts- und Sozi- 
alpolitik bietet. Fragen nach empirischen Puzzles, die Herleitung theoretischer Erwartungen oder die Illustration von Akteursstrategien finden sich nur ansatzweise, so dass bei der Verwendung für Lehrzwecke in fortgeschrittenen Studienabschnitten nach Möglichkeit auch die angegebene Ergänzungsliteratur verwendet werden sollte. Im direkten Vergleich ist das Buch von Pollack u.a. hinsichtlich der Präsentation empirischer Daten und der Anregung eigenständiger Forschungsleistungen stärker, während Puetters Analyse es besser versteht, die zentralen Akteure zu benennen und deren Charakteristika herauszuarbeiten. Ungeachtet dieser unterschiedlichen Schwerpunktsetzung stellen beide Bücher eine lohnenswerte Ergänzung zur vorhandenen Forschungsliteratur dar und können sehr gut in der universitären Lehre eingesetzt werden.

Jale Tosun 